\title{
The tip-of-the-tongue phenomenon: Blocking or partial activation?
}

\author{
ANTJE S. MEYER \\ Max Planck Institute for Psycholinguistics, Nijmegen, The Netherlands \\ and \\ KATHRYN BOCK \\ University of Illinois, Urbana-Champaign, Illinois
}

\begin{abstract}
Tip-of-the-tongue states may represent the momentary unavailability of an otherwise accessible word or the weak activation of an otherwise inaccessible word. In three experiments designed to address these alternative views, subjects attempted to retrieve rare target words from their definitions. The definitions were followed by cues that were related to the targets in sound, by cues that were related in meaning, and by cues that were not related to the targets. Experiment 1 found that compared with unrelated cues, related cue words that were presented immediately after target definitions helped rather than hindered lexical retrieval, and that sound cues were more effective retrieval aids than meaning cues. Experiment 2 replicated these results when cues were presented after an initial target-retrieval attempt. These findings reverse a previous one (Jones, 1989) that was reproduced in Experiment 3 and shown to stem from a small group of unusually difficult target definitions.
\end{abstract}

The familiar tip-of-the-tongue (TOT) experience suggests that recovering a word from the mental lexicon involves something more than a single, simple look-up (Bock, 1987; Garrett, 1988; Levelt, 1989). In addition to identifying a lexical concept that is adequate for conveying an idea, the speaker must retrieve or assemble a word form that denotes the concept. Failures to complete this second step seem to precipitate TOT states. Such failures have therefore been scrutinized with the goal of illuminating the operations of word-form retrieval (A. S. Brown, 1991; Burke, MacKay, Worthley, \& Wade, 1991).

The purpose of the present work was to try to arbitrate some of the TOT-derived evidence for two contrasting hypotheses about the mechanisms of word-form retrieval. Both of them assume an activation-based process, according to which the most highly activated forms are retrieved or assembled, either in production or in recognition. However, they differ over how that activation is achieved. The first hypothesized mechanism is simple activation of tar-

This work was supported in part by research grants from the National Science Foundation (BNS-8617659, 9009611) and the National Institutes of Health (R01-HD21011). We thank Gregory Jones and Joseph Stemberger for their comments on the manuscript, and Laura Carlson, Cooper Cutting, Kathloen Eberhard, Patricia Kaiser, Roger Halley, WooSeoc Hann, Brian Kleiner, Helga Loebell, Carol Miller, and G. A. Radvansky for their assistance in data collection. Patricia Kaiser deserves a special acknowledgment for her help with the data tabulation. Reprint requests may be sent to either author. Antje Meyer is at the Max Planck Institute for Psycholinguistics, Postbus 310, NL-6500 AH Nijmegen, The Netherlands, and Kathryn Bock is at the Department of Psychology, 603 East Daniel St., University of Illinois, Champaign, IL 61820. get forms, including spreading activation among similar word forms, culminating in the selection of the word that has accrued more activation than any other candidate (Collins \& Loftus, 1975; Dell, 1986; MacKay, 1987). The second proposal crucially involves the inhibition of phonologically similar words: As words become activated, they try to suppress their competitors (Grainger, 1990; Stemberger, 1985). The word that wins is the one that retains more activation than its alternatives.

These contrasting proposals can be brought to bear on the explanation of TOT states. On the simple-activation account, the target word may be activated along with a set of related words, but if it is less activated than its relatives, or fails to surpass a retrieval threshold, it may not be generated. However, because the target is in fact activated, it may remain tantalizingly close to retrieval, and some of its relatives may be retrieved in its place. Following A. S. Brown (1991), we will call this the incomplete activation hypothesis (see also Burke et al., 1991, and Yaniv \& Meyer, 1987). Its implication is that the TOT state represents inadequate activation of the target word, and the extraneous words that are sometimes attendant are by-products of normal variations in activation patterns.

The incomplete-activation hypothesis receives indirect support from an array of studies that suggest that speakers are more likely to overcome TOT states when they receive valid phonological cues to the target word than when they receive invalid ones (Brennen, Baguley, Bright, \& Bruce, 1990; Freedman \& Landauer, 1966; Gruneberg \& Monks, 1974; Pease \& Goodglass, 1978). The cues in these studies were the initial letters of the target words, but Kozlowski (1977) obtained similar results by using whole words that rhymed with the targets. 
Mechanisms of suppression or interference bring into play the possibility of a different etiology for TOT states. Such mechanisms are implicated in an intriguing hypothesis formulated by Jones (1989), according to which phonological relationships among words may cause TOTaccompanied retrieval failures. This hypothesis, the blocking hypothesis, was attributed to Woodworth $(1929,1938$; see also Reason \& Lucas, 1983). The essence of the blocking hypothesis is that the similar-sounding words that sometimes come to mind in place of a sought-for target actually obstruct target retrieval, thereby causing the TOT state. Jones dubbed these words interlopers.

Jones (1989; see also Jones \& Langford, 1987, and Maylor, 1990) tested the blocking hypothesis by simulating the speaker's experience of interlopers. The assumption was that if TOT states arise because interlopers interfere with access to the target, it should be possible to precipitate TOT states by intruding words that are phonologically related to a target word into efforts to retrieve that target. To do this, Jones modified a procedure originally used by R. Brown and McNeill (1966), in which subjects were given the definitions of rare words (e.g., "medieval forerunner of chemistry" for the target word alchemy). Each definition was accompanied by a cue word that was related to the target in sound (e.g., axial), by a cue word that was related in meaning, by a cue word that was related in both sound and meaning, and by a cue word that was not related to the target word. As predicted by the blocking hypothesis, TOT states were more frequent when cue and target were phonologically related than when they were related only in meaning or were unrelated.

These studies are consistent with a lexical selection mechanism that can have blocking as its consequence, and appear incompatible with findings that suggest that phonologically related words may aid in overcoming TOT states (Kozlowski, 1977). However, because the question addressed in the Jones (1989; Jones \& Langford, 1987) studies was specific to the effect of interlopers on the incidence of TOT experiences and not on the incidence of successful target retrieval, the data from those experiments cannot be unambiguously brought to bear on the broader issue of the nature of the selection mechanism. Since correct retrievals were not reported, the findings do not reveal whether the TOT states were positively or negatively correlated with the accessibility of the targets. Consequently, the results are equally consistent with the incomplete-activation hypothesis.

The above conclusion is true for the following reason: Suppose that all of the subjects who did not report TOT states on any given trial (non-TOT subjects) fell into just one of two groups-those who knew the target and those who did not. If, in fact, all of the non-TOT subjects knew the target word, we would be justified in assuming that a higher incidence of TOT states reflected decreased target accessibility. However, it is also possible that none of the non-TOT subjects knew the target word. In this case, a TOT state could be regarded as a reflection of in- creased target accessibility. The true situation is almost certainly somewhere between these extremes, with the non-TOT reports divided into successful and unsuccessful target retrievals. Depending on their balance, the TOT reports themselves take on different implications for the lexical selection mechanism.

In an effort to assess these implications, we carried out a set of TOT-induction experiments similar to those reported by Jones (1989; Jones \& Langford, 1987). In them, we recorded the frequencies of correct and incorrect responses along with the frequencies of TOT states. The subjects in all of the experiments were presented with definitions and were asked to report the target words when they knew them. The definitions were accompanied by cues that were related to the targets in sound alone, by cues that were related in meaning alone, and by cues that were not related to the targets.

An important modification of the procedure concerned the assignments of definitions to cuing conditions. The previous studies used different sets of definitions in each cuing condition. To ensure that variations among the definitions did not contribute to the cuing effects, in Experiments 1 and 2 we presented each item once in every cuing condition, although individual subjects received each item just once. Because of the difficulty of finding words that were both semantically and phonologically related to all of the targets, we omitted this compound condition in Experiments 1 and 2. However, in Experiment 3, we included such cues and employed the same design as did Jones (1989), using a different set of definitions in each of the four experimental conditions. The definitions were the same as those used by Jones and were assigned to the same conditions as those used in his experiment.

For correct target retrievals, the experimental outcomes that are predicted from simple-activation and suppression views of lexical selection differ in the obvious way. If the increase in TOT reports that accompanied phonological cuing in previous work was a product of a simple activation mechanism (i.e., if phonological cues increased target accessibility), phonological cues should be more likely to elicit correct target reports than should unrelated cues. However, if the retrieval process includes a suppression component (i.e., if phonological cues decrease target accessibility), phonological cuing should decrease the number of correct target reports relative to unrelated cues.

Because correct responses and TOT reports may not be fully independent measures, the predictions for TOT reports are less straightforward. They depend in part on assumptions about the representation and access of lexical knowledge. If knowledge of the target words varies in a continuous fashion (and is very broadly distributed across our subjects), the simple-activation hypothesis predicts an increase in TOT reports relative to unrelated cues. The argument would be that subjects who have too little knowledge of a target word to ever report it accurately might nonetheless achieve a TOT state, given the proper cue. With this continuity assumption, the suppression hypothesis likewise predicts an increase in TOT re- 
ports relative to unrelated cues, putting the burden of proof on the correct responses.

The situation becomes more complicated if lexical knowledge is represented or accessed more discretely, so that subjects either know the word or do not. In these circumstances, TOT reports will simply vary inversely with correct target reports. The simple-activation hypothesis predicts that, relative to the unrelated condition, cued correct retrievals should increase as TOTs decrease, whereas the suppression hypothesis predicts that cued correct retrievals should decrease as TOTs increase. We will refer to this as the discrete assumption.

To determine which of these assumptions about the distribution of lexical knowledge is more nearly right, in Experiments 1 and 2 we examined the total numbers of responses that could reasonably be taken to reflect word knowledge (correct and TOT responses combined) as percentages of possible responses. ${ }^{1}$ Given large subject samples (there were 411 subjects in Experiment 1 and 348 in Experiment 2), stability in these percentages across cuing conditions and across experiments would be consistent with discrete representation or accessibility of target knowledge.

Because the blocking hypothesis applies specifically to phonologically related words, another issue is whether phonological cuing behaves in a qualitatively different way than does semantic cuing. In Jones's experiments (1989; Jones \& Langford, 1987), semantic cues elicited no more TOTs than did the unrelated cues, which is consistent with the argument that blocking is a sound-related phenomenon. To examine this further, all of the experiments reported below compared semantic and phonological cues for the target words.

\section{EXPERIMENT 1}

In the first experiment we adapted the procedure employed by Jones (1989) in order to assess the incidence of correct target reports along with TOT states, with the modified experimental design described in the introduction. We employed the version of his procedure in which cues followed rather than preceded the target-word definitions, because the phonological blocking effect that Jones (1989) obtained was more powerful when the cues followed.

\section{Method}

Subjects. There were 411 subjects in Experiment 1. In all three experiments, the subjects were undergraduates at Michigan State University who participated in return for extra credit in introductory psychology courses. The experiments were conducted during different academic years, making it unlikely that any student participated more than once.

Materials. The materials included 36 target-word definitions that were taken over and, in 6 cases, slightly modified from the materials listed by Jones (1989). Most of the modifications were designed to make the definitions and targets more compatible with English usage in the United States. The changes are noted in the list of materials given in Appendix A (the list does not indicate spelling differences between the two sets of materials but adheres to
United States' conventions). Four of the definitions from Jones's unrelated and both semantically and phonologically related cuing conditions were eliminated at random to create an item set appropriate to the design of the present experiment and suitable for the available testing time.

Each target word was matched with three single-word cuesone a phonologically related cue, another a semantically related cue, and the third an unrelated cue. The cues were in most cases different from those of Jones (1989) but had the same features. The phonological cues had the same initial sound and letter, the same number of syllables, and the same stress pattern as did the corresponding targets but were unrelated to them in meaning. The semantic cues were related to the targets in meaning but not in form and were selected from thesaurus and dictionary listings for the target words (Roget's International Thesaurus, 1977; Roget's Thesaurus of English Words and Phrases, 1982; Webster's Collegiate Thesaurus, 1976); Webster's New Collegiate Dictionary, 1981). The selected words differed from the targets in initial sound and letter, number of syllables, and stress pattern, and, in all but three cases, in the vowel in the stressed syllable. The unrelated cues were unrelated to the targets in both meaning and form, differing from them in number of syllables and in stress pattern, and sharing no phonological segments with them.

We did not adopt Jones's (1989) cues in most of the cases in which that would have been possible, but attempted to find new cues to better establish the generality of the cuing or blocking effects. However, in 4 cases, the best available cues were identical to Jones's (these are starred in Appendix A). They met the same criteria as the remaining 104 cues, which were newly selected. The frequencies of the newly selected cues were, on the average, higher than those used in Jones's experiments.

Three 36-item lists were created from pairings of the target definitions with these cues. Each list contained all 36 definitions and an equal number of phonological, semantic, and unrelated cues (12 of each), 1 cue for each definition. Across the three lists, each definition was paired once with each of its 3 target cues.

The same pseudorandom order of definitions was used in all three lists. The cue order was constrained so that there were no more than two consecutive occurrences of the same cue type.

Each list began with a single practice item. The practice definition (for the target sextant) was "An instrument used for measuring angular distances, used especially in navigation to observe the altitudes of celestial bodies." The cue that accompanied this definition was the word latitude.

The cues for the targets on each list were assembled into individual test booklets, with different booklets for each of the three lists. Every booklet contained a single page of instructions followed by 37 single-page response sheets ( 1 for each test item, including the practice item). The response sheet contained the cue word at the top and a space for writing the target if, after reading the cue, the subject knew the word. If the target word was unknown, the subject responded to a series of questions about TOT states. The questions asked whether the subject was in a TOT state and, if so, requested best guesses about the initial letter and the number of syllables contained in the target word.

Procedure. All subjects were tested together in a large auditorium. They were randomiy assigned to one of the three cue lists, with the constraint that an equal number of subjects received each list.

At the beginning of the experiment, each subject received a test booklet. The experimenter read aloud the printed instructions (see Appendix B), which described TOT states and explained the experimental trials and the procedure for filling in the response sheets. The experimenter then read the first definition aloud, instructed the subjects to turn to the page containing the cue word, and then repeated the definition. The subjects were allowed $50 \mathrm{sec}$ to fill out the response sheet. At the end of this period, the next defini- 
tion was presented. This procedure was repeated for each of the 36 words in the list.

Response coding and data analyses. The response words were classified either as targets or as nontargets. Target responses included all correctly spelled target words as well as misspelled target words, provided that the target word was the most likely pronunciation (following regular spelling-to-sound correspondences). All other responses were treated as nontargets.

On 16 trials, the subjects gave response words and also claimed to be in TOT states. The response words were classified as targets or nontargets, but the TOT responses were not included in the count of TOT states. On 12 trials, the subjects failed to respond in any way. These cases were treated as missing values and omitted from the analyses.

Analyses of variance (ANOVAs) treating subjects $\left(F_{1}\right)$ and items $\left(F_{2}\right)$ as random factors were carried out separately on the correct responses and the TOT reports. Effects that are reported as significant were reliable at or beyond the .05 level. Planned pairwise comparisons among the cuing conditions were carried out by constructing $95 \%$ confidence intervals with two-tailed test statistics (Kirk, 1968).

\section{Results}

Table 1 shows the distributions of correct target responses and TOT reports for the three cuing conditions.

The cues varied reliably in their effect on correct target retrieval $\left[F_{1}(2,820)=4.71 ; F_{2}(2,70)=6.20\right]$. Phonological cues elicited more correct targets than did either semantic or unrelated cues, and semantic cues elicited more correct targets than did unrelated cues (the confidence intervals were 31 for subjects and 28 for items).

For TOT states, although the overall ANOVA was not significant $\left[F_{1}(2,820)=1.47 ; F_{2}(2,70)=0.94\right]$, the planned comparisons revealed that there were reliable differences among the cues. There were significantly more TOTs after semantic than after phonological cues. After phonological cues, the number of TOTs fell just one response short of a conventionally significant difference with the unrelated condition in the subjects analysis, with fewer TOTs after phonological cues than after unrelated cues. The semantic cues did not differ from the unrelated cues (the confidence intervals were 31 for subjects and 39 for items).

The percentages of responses within each condition that reflected knowledge of the target (summing the correct responses and the TOT reports) are shown in the third column of Table 1 . The percentages are obviously very similar for all conditions, with roughly one-third of the responses being either a correct response or a TOT re- port, regardless of cue type. ANOVAs on the summed responses found no significant differences for subjects or items $\left[F_{1}(2,820)=1.49 ; F_{2}(2,70)=1.36\right]$.

Nontarget responses occurred about equally often in the three conditions, $34 \%$ of the time overall. There were 1,688 nontarget responses in the phonological condition, 1,691 in the semantic condition, and 1,656 in the unrelated condition.

We examined the distribution over cuing conditions of correct specifications of initial letters and numbers of syllables. There were more correct first letters and more correct syllable estimates in the phonological condition than in the semantic and unrelated conditions $(184,146$, and 134 for first letters and 209, 187, and 174 for syllables, respectively). This pattern suggests that phonological cues heightened the accessibility of target word features, but further inspection of the data undermined this conclusion. In the semantic and unrelated conditions, the subjects frequently responded with the first letter and the number of syllables of the cue word (note that in these conditions, but not in the phonological condition, the first letters and numbers of syllables of the cues differed from the targets). In these two conditions, there were 96 and 73 cuebased first-letter responses and 222 and 79 cue-based syllable estimates, respectively.

To estimate the strength of cue-based guessing relative to correct responding in the phonological condition, we calculated the proportion of cue-based guesses within the set of correct first letters and cue-based guesses combined over the semantic and unrelated conditions. On the average, .52 of the first-letter responses and .47 of the syllable estimates were cue-based guesses. If we assume a comparatively strong tendency to make cue-based guesses in the phonological condition, and deduct .52 of the target first-letter specifications and .47 of the target-syllable estimates from the totals, the appearance of greater target knowledge in the phonological condition vanishes. After these adjustments, there remained 115 accurate firstletter responses and 114 accurate syllable estimates in the phonological condition. Taking these adjustments into account and expressing the response tendencies as percentages of the number of TOT states in each cue condition, there were $18 \%, 21 \%$, and $20 \%$ correct first letters and $18 \%, 27 \%$, and $26 \%$ correct syllable estimates in the phonological, semantic, and unrelated conditions, respectively.

Table 1

Distribution of Responses Across Cuing Conditions in Experiment 1

\begin{tabular}{cccc}
\hline & \multicolumn{3}{c}{ Response Category } \\
\cline { 2 - 4 } Cuing Condition & $\begin{array}{c}\text { Number of Correct } \\
\text { Targets }\end{array}$ & $\begin{array}{c}\text { Number of TOT } \\
\text { States }\end{array}$ & $\begin{array}{c}\text { Targets and TOTs as } \\
\% \text { of Total Responses } \\
\text { Within Condition }\end{array}$ \\
\hline Phonological & 1,013 & 628 & 33.3 \\
Semantic & 951 & 682 & 33.1 \\
Unrelated & 917 & 658 & 31.9 \\
Mean \% & 19.5 & 13.3 & \\
\hline
\end{tabular}

Note-Mean percentages were calculated excluding missing responses. 
We report similarly corrected data derived from the letter and syllable responses in the subsequent experiments, in which comparable guessing patterns appeared. However, because of the tenuousness of the inferences that can be drawn from these data, we do not discuss them further.

\section{Discussion}

The results indicated that cuing target words with phonologically similar words helps rather than hinders their retrieval. The subjects were more likely to retrieve defined words when they were given phonological cues than when they were given semantic or unrelated cues. These results point toward simple activation rather than suppression in lexical selection, and toward a partialactivation account of TOT states rather than a blocking account.

The TOT reports themselves cannot be interpreted independently of the correct target reports, for reasons that we elaborated in the introduction. In the present experiment, the incidence of those reports after phonological cues was inversely related to the incidence of correct target reports, with fewer TOTs after phonological cues than after unrelated cues. This difference was only marginally significant, but together with the significant effect for correct responses, this pattern is also more consistent with activation than with suppression.

Semantic cues yielded fewer correct responses and more TOTs than did phonological cues. Relative to unrelated cues, the semantic cues increased the number of correct responses and also increased the number of TOTs, but not reliably so. Although this pattern is slightly different from the phonological pattern, it does not diverge in a way that suggests that the underlying dynamics of selection are qualitatively different.

Whether viewed from the perspective of the correct target or TOT reports, the results are different from those of Jones (1989; Jones \& Langford, 1987). A possible explanation for the discrepancy between our results and Jones's may be found in differences in baseline responding within the cuing conditions employed by Jones. Experiment 2 begins to address that possibility.

\section{EXPERIMENT 2}

Experiment 2 had two goals. One was to assess how often the subjects could retrieve each of the targets on the basis of its definition alone. To accomplish this, the definitions in this experiment were immediately followed by an opportunity for the subjects to write down the target word if they knew it.

The second goal was to further examine the effect of cuing on correct target retrieval, in order to replicate the results of Experiment 1. Accordingly, a cue word was made available, but only after the initial response opportunity, permitting examination of the effects of cuing in just those cases when uncued retrieval failed. Because subjects who have failed to retrieve the target word at the point of cue presentation should be in states ranging from no target knowledge at all to TOT experiences, and because this range is a truncated version of the range that directly follows the presentation of a target definition, the effects of cues at this point should be informative about their role in promoting or hindering correct target retrieval.

Obviously, phonological cues presented at this point have less chance of inducing TOT states in the manner predicted by the blocking hypothesis. However, they could still decrease the likelihood of resolving an existing TOT, thereby increasing the incidence of such states and decreasing the likelihood of correct retrievals relative to unrelated cues. Alternatively, the cues could function as they did in Experiment 1, serving to increase correct retrievals and decrease TOTs.

\section{Method}

Subjects. There were 348 subjects from the same source as in Experiment 1.

Materials. The materials were the same as in Experiment 1 except for the arrangement of the response booklets. The response sheet for each item consisted of two pages, with the first page containing the definition of the target word and a space for writing the word down if the subject knew it. If the target word was unknown, the subject was directed to turn to the second page of the response sheet. The second page was the same as the response sheet in Experiment 1 , with the cue at the top.

Procedure. The procedure was in most respects the same as in Experiment 1 . The instructions were modified slightly to reflect the booklet changes. To encourage the subjects to pay attention to the cue words on the second response page, the instructions included a sentence that said that the cue "may help you to think of the word. $" 2$ The experimenter read each definition aloud just once and then waited for $50 \mathrm{sec}$ while subjects filled out the response sheets.

Response coding and data analyses. The responses were coded according to the same criteria used in Experiment 1. There were 7 instances in which subjects wrote down response words but also claimed to be in a TOT state. These were counted as correct or incorrect responses and were not treated as TOT events. There were 336 missing values ( $2.7 \%$ of all cells) from trials in which subjects failed to respond in any way.

The data analyses were the same as in Experiment 1. Correct responses were analyzed separately as either before-cue or after-cue responses.

\section{Results}

Table 2 shows the distributions of correct before-cue and after-cue target responses and TOT reports for the three cuing conditions.

The number of correct responses made before the cues were presented did not differ significantly across the three conditions $\left[F_{1}(2,694)=0.82 ; F_{2}(2,70)=1.03\right]$. After the cues were presented, however, the distribution of correct responses varied with cue type $\left[F_{1}(2,694)=28.10\right.$; $\left.F_{2}(2,70)=12.52\right]$. As Table 2 indicates, correct responses were far more likely after phonological cues than after cues of the other two types, and semantic cues also reliably increased the number of correct target reports (the confidence intervals were 10 for subjects and 16 for items).

The third column of Table 2 gives the number of reports of TOT states across conditions. Although the con- 
Table 2

Distribution of Responses Across Cuing Conditions in Experiment 2

\begin{tabular}{ccccc}
\hline & \multicolumn{4}{c}{ Response Category } \\
\cline { 2 - 5 } Cuing Condition & $\begin{array}{c}\text { Number of Correct } \\
\text { Targets Reported } \\
\text { Before Cue }\end{array}$ & $\begin{array}{c}\text { Number of Correct } \\
\text { Targets Reported } \\
\text { After Cue }\end{array}$ & $\begin{array}{c}\text { Number of TOT } \\
\text { States }\end{array}$ & $\begin{array}{c}\text { Targets and TOTs as } \\
\text { \% of Total Responses } \\
\text { Within Condition }\end{array}$ \\
\hline Phonological & 697 & 108 & 578 & 33.1 \\
Semantic & 701 & 49 & 635 & 33.2 \\
Unrelated & 667 & 28 & 564 & 30.1 \\
Mean \% & 16.5 & 1.5 & 14.6 & \\
\hline
\end{tabular}

Note-Mean percentages were calculated excluding missing responses.

dition differences were not significant in the ANOVA $\left[F_{1}(2,694)=2.97 ; F_{2}(2,70)=2.92\right]$, the planned comparisons indicated that there were reliably more TOT reports after semantic cues than after either phonological or unrelated cues. The latter two cue types did not differ (the confidence intervals for these comparisons were $\mathbf{3 0}$ for subjects and 31 for items).

Correct targets (both before and after the cue) or TOT states occurred on roughly a third of the trials in each condition, as shown in the fourth column of Table 2 . There was a difference of about $3 \%$ between the unrelated condition and each of the other conditions, which produced a significant effect in ANOVAs on the summed responses $\left[F_{1}(2,694)=6.43 ; F_{2}(2,70)=7.93\right]$.

There were 2,909 nontarget responses in the beforecue period. After the cues, there were 300,377 , and 235 nontarget responses in the phonological, semantic, and unrelated conditions, respectively.

The first-letter specifications and syllable estimates were corrected and converted to percentages of the TOT responses in the manner described in Experiment 1. Across the phonological, semantic, and unrelated conditions, the corrected percentages of accurate responses were $16 \%$, $17 \%$, and $15 \%$ for first letters and $21 \%, 26 \%$, and $15 \%$ for number of syllables, respectively.

\section{Discussion}

In the after-cue responses, the phonological cues again failed to produce effects consistent with blocking. For after-cue correct target reports, the phonological cues were more effective than either semantic or unrelated cues. Although they produced somewhat more TOT reports than the unrelated cues, the difference was small and unreliable and, in the face of the strong positive effect for the correct targets, difficult to reconcile with a suppression account of lexical selection and the companion blocking account of TOTs. Recalling the continuity assumption about target knowledge outlined in the introduction, the occurrence of more correct responses along with more TOTs is indicative of retrieval support rather than of suppression.

The semantic cues followed the same general trends as did the phonological cues in eliciting correct responses and TOTs, although they had a stronger and more reliable impact on TOTs. Still, they did not behave in a fashion that was qualitatively different from that of the phonological cues.

The chief difference between this and the foregoing experiment is the appearance of an altered pattern of evidence for facilitation of target retrieval by phonological cues (relative to unrelated cues). The present pattern is more in line with continuity than with discreteness of representation. This may be an indirect consequence of the "last resort" status of the cues in the present experiment: They were used only when initial retrieval attempts failed. It is notable that in one respect, the target knowledge totals are also different from those in Experiment 1. There was a reliable related-cue effect in the summed correct and TOT responses, with semantic and phonological cues more often associated with either correct responses or TOTs than were unrelated cues. However, contrasting these totals to those in Experiment 1 (compare the last columns of Tables 1 and 2), it is evident that the difference is entirely attributable to the unrelated condition. For some reason, the subjects in the unrelated condition failed to access word knowledge that seemed to be available in both of the related conditions in this experiment and in all of the conditions of Experiment 1.

In the before-cue responses, there was no evidence of differences among the cuing conditions, as one would expect. However, it is possible to use these data to examine, at least in a preliminary way, whether or not the groups of targets that were assigned to the four cuing conditions of Jones's experiments differed in retrievability for our subjects. For the definitions that appeared in Jones's (1989) phonological, semantic, both semantically and phonologically related, and unrelated conditions, there were means of $43.5 \%, 53.7 \%, 62.6 \%$, and $74.0 \%$ correct target word reports, respectively. Although the last two of these percentages are based on subsets of the items from the Jones experiment (8 of 10 in both cases), the differences are suggestive. Since smaller numbers of beforecue retrievals indicate greater difficulty in recovering words on the basis of their definitions alone, it appears that the items in Jones's (1989) phonological condition may have been the most difficult.

These results diverge from those of Maylor (1990), who presented the definitions from the Jones (1989) experiments to 7 subjects without any cues. She reported grouped means for the words from the unrelated and 
phonological conditions and for the words from the semantic and the both semantic and phonological conditions. These means indicated better retrieval of the words in the former two sets than in the latter two sets. Similar groupings of our data yield roughly equal retrieval levels. Maylor also reported that there was no discernible difference between definitions cued with phonologically related words and those cued with phonologically unrelated words (comparing the words in the phonological and in the both semantic and phonological conditions with those in the semantic and unrelated conditions). This is again very different from our before-cue retrieval results. Since an obvious source for these differences is in subject samples (Maylor's subjects were older and, as were Jones's, British), it is important to see whether Jones's results are replicable in the circumstances of our experiments, in which we used his assignments of items to cuing conditions. That was the purpose of Experiment 3.

\section{EXPERIMENT 3}

Experiments 1 and 2 both suggested that phonological cues facilitated word retrieval more than did unrelated cues. In an effort to explain why these results diverge from those of similar studies (Jones, 1989; Jones \& Langford, 1987), we examined a difference in the experimental designs. In Experiments 1 and 2, every definition occurred in every cue condition. In the Jones studies, however, a different set of definitions appeared in each cue condition, so unanticipated differences among the definitions in TOT susceptibility may have affected the condition differences. The before-cue results of our Experiment 2 tend to support this conjecture.

To examine this further, in Experiment 3 we assigned the definitions to the same conditions in which they had appeared in Jones's (1989) experiment. We also added Jones's fourth cuing condition, in which the cues were both semantically and phonologically related to the target words, as part of an effort to replicate as many features of the earlier work as was feasible while retaining important components of the design of Experiments 1 and 2. Most important, we again asked the subjects to report the target words when they believed they knew them.

\section{Method}

Subjects. There were 261 subjects from the same source as in the previous experiments.

Materials. The materials from Experiments 1 and 2 were modified by adding 4 definitions and 12 cues, originally employed by Jones (1989), to create a set of $\mathbf{4 0}$ definition-plus-cue pairs. The 4 new definitions are shown at the end of Appendix A. The 12 new cues included 2 unrelated cues (for 2 of the added definitions in Appendix A) and 10 cues that were both semantically and phonologically related to their targets, as given in Jones (1989). These cues, which we will call both cues, and their respective targets were abnormality-anachronism, bemock-bemuse, binomial-bilateral, disclaim-default, fervent-flippant, gelding-gosling, hepatichemorrhage, hibiscus-herbacious, injudicious-iniquitous, and loquacious-literate (note that we used disclaim in place of disclaimer and fervent in place of fervency, in line with the modifications shown in Appendix A).

For the remaining 28 items, we used only the cues appropriate to the conditions in which the corresponding 28 definitions had appeared in Jones's (1989) experiment. The condition assignments of the items in Jones's study are indicated in Appendix A. Except for the both cues and the two new unrelated cues, all the cues were selected from those used in Experiments 1 and 2

A single list of $\mathbf{4 0}$ items was developed from these materials. The order of the items in the list was constrained so that in each successive group of 4 items there was 1 item from each of the four cue conditions, and the average serial position of items of each of the four types was the same. The order was otherwise random. The list began with the same practice item used in the previous experiments, so that the subjects received a total of 41 definitions.

The response booklets for the experiment contained neither the definitions nor the cues for the target words. The one-page response sheet for each item contained a space for writing the target word followed by the questions about TOT states used in the previous experiments.

Procedure. The procedure in most respects duplicated that of Jones (1989). The experimenter read each target-word definition, tapped the microphone, and then pronounced the cue word. After $4 \mathrm{sec}$, this sequence was repeated. Following a 30 -sec response period, the next definition and cue were read in the same fashion, continuing through the 41 -item list. The instructions were modified from the previous experiments to reflect these procedural changes.

Response coding and data analyses. Responses were coded and analyzed as in the previous experiments. There were 33 trials on which the subjects wrote down a response word and simultaneously claimed to be in a TOT state, and 2 missing trials.

\section{Results}

The data are displayed in Table 3.

For correct target responses, there was a reliable effect of cue type for subjects $\left[F_{1}(3,780)=50.41\right]$ but not for items $\left[F_{2}(3,36)=0.17\right]$. In planned comparisons of the subject means, the unrelated cues elicited significantly more correct targets than did any other cue type. The phonological and both cues elicited the fewest and did not differ from each other. Semantic cues fell in between, differing from all the others. The confidence intervals for these comparisons were 18 for subjects and 328 for items.

For TOTs, there was also a reliable effect of cue type for subjects $\left[F_{1}(3,780)=29.57\right]$ but not for items $\left[F_{2}(3,36)=2.34, p<.10\right]$. Planned comparisons of the subject means showed that both cues were associated with the most TOTs, followed in order by phonological and unrelated cues, which did not differ, and semantic cues, which elicited significantly fewer TOTs than did any other cue type. For these comparisons, the confidence intervals were 24 for subjects and 87 for items.

Incorrect target reports constituted 3,073 of all responses $(29.4 \%)$. There were 759 after phonological cues, 976 after semantic cues, 634 after both cues, and 704 after unrelated cues.

The first-letter guesses and syllable-number estimates were corrected and converted to percentages of the reported TOT states. For the phonological, semantic, unrelated, and both cuing conditions, the respective per- 
Table 3

Distribution of Responses Across Cuing Conditions in Experiment 3

\begin{tabular}{lcc}
\hline Cuing Condition & \multicolumn{2}{c}{ Response Category } \\
\cline { 2 - 3 } \multicolumn{1}{c}{$\begin{array}{c}\text { Number of Correct } \\
\text { Targets }\end{array}$} & $\begin{array}{c}\text { Number of TOT } \\
\text { States }\end{array}$ \\
\hline Phonological & 375 & 402 \\
Semantic & 434 & 277 \\
Both semantic and phonological & 388 & 504 \\
Unrelated & 577 & 392 \\
Mean \% & 17.0 & 15.1 \\
\hline
\end{tabular}

Note-Mean percentages were calculated excluding missing responses.

centages of accurate first-letter guesses were $14 \%, 14 \%$, $13 \%$, and $16 \%$, and of accurate syllable estimates, $13 \%$, $30 \%, 34 \%$, and $26 \%$.

\section{Discussion}

The findings of Experiment 3 dovetail in important respects with those of Jones (1989). The definitions in the phonological cuing condition, the same ones used in Jones's phonological cuing condition, were the least likely to elicit correct target retrievals and, next to the items in the both condition, the second most likely to elicit TOT states. This is exactly the pattern predicted by the suppression hypothesis of lexical selection, and the appearance is that phonological cues blocked target retrieval and induced TOT states. However, the same findings diverge from those of Experiments 1 and 2, in which we found that phonological cues were the most likely to elicit correct target retrievals and the least likely to be associated with TOT states. Since the major difference in the previous experiments was that all of the items appeared in every cuing condition, it seems that the subset of words from Jones's (1989) phonological condition is particularly vulnerable to retrieval problems.

There are two features of the items that may have been responsible for the difficulty-either the target words or the definitions for them. Although it is impossible to convincingly distinguish these alternatives with the data available to us, on one important measure of word familiarity, the words in the phonological condition did not differ from those in the other three conditions. Jones (1989) sampled all of the targets from the same Thorndike-Lorge frequency category, and we verified their frequencies in the CELEX database (Burnage, 1990), which includes nearly 18 million words of English text. The numbers of occurrences per million for the words in the phonological, semantic, unrelated, and both conditions were $1.8,0.5,1.0$, and 0.8 , respectively. These frequencies suggest that, if anything, the phonological targets may be more familiar than the other three types, but the differences are not large.

There are, of course, other features of words that may make them hard to retrieve, including meaning complexities that could also make them hard to define. If that is the problem with the words in Jones's (1989) phonological condition, it would readily explain why the definitions for those words were less evocative, on balance, than the definitions for the words in the other categories in the before-cue retrievals in Experiment 2.

Paradoxically, the phonological cues are the least likely culprits for the problems with the words in the phonological condition. There are two reasons. First, most of the cues in the phonological condition in Experiment 3 were different from those of Jones (1989), yet they yielded similar results. Second, the before-cue retrievals in Experiment 2 necessarily reflect cue-independent factors, but they, too, revealed the difficulty of retrieving the words from the phonologically cued subset of the Jones items.

This is not to say that the cues were irrelevant to responding. The differences observed among the conditions in Experiments 1 and 2 obviously must be attributed to differences in the effects of the cues. Likewise, in Experiment 3 , there were differences among the conditions that cannot be explained solely in terms of variations in the difficulty of the target words. The words from the both condition represent a clear case: There were relatively many uncued correct target reports for these words in Experiment 2, yet relatively few cued correct target reports and relatively many TOTs in Experiment 3. Unfortunately, because this condition could not be included in Experiments 1 and 2, we cannot say with any confidence what the contribution of the both cues might be.

Cue effects may also be responsible for some of the differences between the results of Experiment 3 and those of Jones (1989). Of our TOT reports, 32\% occurred in the both condition, $26 \%$ in the phonological condition, $25 \%$ in the unrelated condition, and $18 \%$ in the semantic condition. In Jones (1989), $27 \%$ of the TOT reports occurred in the both condition, $30 \%$ in the phonological condition, $21 \%$ in the unrelated condition, and $21 \%$ in the semantic condition. Disparities among the cues may have something to do with these differences in the incidence of TOTs. The cue disparities are most obvious in the unrelated condition. In our experiment, but not in Jones's, 7 of the 10 unrelated cues were monosyllables, and in that respect were very unlike the cues in the other conditions (which were, with just one exception, polysyllabic). The subjects may have guessed that these cues had little to do with the targets and may have been less motivated to try to retrieve the corresponding target words.

Despite these differences in TOT results, when correct target responses are also taken into account, very similar retrieval patterns for the four subsets of words emerge 
from the two experiments. As just noted, the TOT reports from Jones (1989) suggest that the hardest-to-recall words were those in the phonologically cued condition, followed in order by the words in the both, unrelated, and semantic conditions. Among correct target responses, we also found that the hardest-to-recall words were those from the phonologically cued condition, followed in order by the words from the both, semantic, and unrelated conditions. Evidently, the phonologically cued words were the most resistant to retrieval. But, considered in light of the findings in Experiments 1 and 2, this is more probably a consequence of the words themselves than of the cues used to bias their retrieval.

\section{GENERAL DISCUSSION}

The goal of this research was to evaluate two explanations for TOT states derived from alternative proposals about a basic mechanism of lexical retrieval. The partialactivation hypothesis suggests that TOT states arise when heightened levels of activation fall short of an adequate level for selection. The suppression hypothesis, in contrast, proposes that TOT states arise when the activation of a word is dampened by its competitors.

The experiments examined how the retrieval of words from definitions was affected by the provision of supplementary cues that were semantically related, phonologically related, or unrelated to the target words. The focus was on the effects of phonological relationships, because they have been implicated in a particular type of TOT-eliciting suppression called phonological blocking (Jones, 1989). Lexical retrieval was indexed in two ways-by the incidence of correct target retrievals and by the incidence of TOT states. In Experiments 1 and 2, targets were correctly retrieved substantially more often when they were cued by phonologically related words than when they were cued by unrelated words. In Experiment 1, TOT states occurred less frequently after phonological than after unrelated cues, whereas in Experiment 2, they were narrowly, but not reliably, more frequent.

In Experiments 1 and 2, semantically cued correct and TOT responses were not distributed in a notably different way from the phonologically cued responses. There were more correct responses following semantic as well as phonological cues in both experiments, and the TOT reports were in the same direction in Experiment 2. Only in the TOT reports in Experiment 1 did the semantic cues behave differently from the phonological cues. However, even this difference was not consistent with the predictions of the suppression hypothesis.

Overall, these findings are more consistent with partial activation than with suppression, both for phonological and semantic relationships. Models of lexical retrieval that incorporate only simple activation of lexical entries predict the superiority of related cues over unrelated cues in the elicitation of correct target retrievals, whether with respect to activation spreading directly between related words (e.g., Collins \& Loftus, 1975) or indirectly from information shared by the cue and the target (e.g., Dell, 1986), or with respect to retrieval enhancement from compound cues (Ratcliff \& McKoon, 1988). Except in the heavily qualified results of Experiment 3, there was no evidence among the correct responses that the provision of semantically or phonologically related information reduced the accessibility of the target words, as a suppression hypothesis would predict.

The support for the partial-activation account from the TOT results is not as direct as that from the correct responses. A straightforward prediction from partial activation is that both correct and TOT responses should increase after cuing. However, as we noted in the introduction, this prediction assumes that TOT reports can be elicited from subjects with too little knowledge of the target word to ever be able to report it. For such a prediction to be realized, the requirement is that knowledge of target words be distributed in such a fashion that, even with cuing, substantial numbers of subjects with partial knowledge of the target word will fail either to retrieve it or to experience a TOT state. In consequence, variations in cue effectiveness should be associated with variable estimates of target knowledge.

Our data, most clearly in Experiment 1, were at odds with this idea. Experiment 1 showed that the level of target knowledge accessible to cuing was extremely stable. In all of the conditions in Experiment 1, and in two of the three conditions in Experiment 2, the subjects gave some evidence of knowing the target words on one-third of the trials. This suggests that when the subjects who correctly retrieved the target word were eliminated, the number of subjects who were candidates for TOT reports was severely restricted. Consequently, the TOT results that are most clearly in line with the partial-activation hypothesis are those in which TOTs occurred less often after related than after unrelated cues, as they did after the phonological cues in Experiment 1.

In both experiments, phonological cues appeared to be superior to semantic cues, eliciting more correct retrievals and fewer TOT states. This is most likely to be due to the supplementary information that phonological cues provide. Semantic cues offer little information about the target words beyond what the definition itself contains, whereas phonological cues provide information about how the target words sound (see Rubin \& Wallace, 1989). Alternatively, the relationship of the semantic cues to the target lexical concepts may simply have been weaker than the relationship of the phonological cues to the target word forms.

The results of Experiment 3 differed substantially from those of Experiments 1 and 2. In Experiment 3, each target definition was assigned to only one condition, and the assignments duplicated those of Jones (1989). With this change, the phonological cues yielded the fewest correct responses and the most TOT states. Although consistent with a phonological suppression mechanism of the sort proposed in the blocking hypothesis (Jones, 1989), this result is more likely a consequence of the subset of words 
that appeared in the phonological condition in Experiment 3 and in Jones's experiment. A post hoc analysis of the before-cue target responses in Experiment 2 showed that this subset of words was the least likely to be retrieved on the basis of definition alone, in the absence of any cue effects. These words-or their definitions-were unusually difficult. Another apparent consequence of this variability among the definitions was that the item effects were rarely significant.

Our findings challenge the interpretation of previously reported evidence for the blocking hypothesis. The present results suggest that phonologically related cue words support the correct retrieval of rare word targets and do so in a way that is not qualitatively different from that of semantically related cues. This is more consistent with a partial-activation account than with a suppression account of TOT states and, in turn, more consistent with simple-activation mechanisms of lexical retrieval.

Still, there are reasons to be cautious about these conclusions. As in previous TOT induction studies, the effects of related cues were assessed relative to an unrelated-cue condition rather than to a no-cue condition. The latter arguably offers a more appropriate baseline for the assessment of retrieval facilitation and suppression but has the disadvantage of eliminating not only the informational value of the cue, but also its motivational value. The choice of an appropriate neutral condition, here as elsewhere (Jonides \& Mack, 1984), is vexed.

Equally serious are concerns about the paradigm itself. Although the results of previous experiments that used the cuing or blocking paradigm have been brought to bear on arguments about lexical selection, the paradigm has features that may prevent it from capturing such processes. Even when cues directly follow definitions, they may appear well after lexical selection has occurred and with correspondingly little opportunity to influence it, having their effects instead on more strategic retrieval efforts. The similarities in the cuing results of Experiments 1 and 2 lend weight to this possibility and invite further scrutiny of the relationship between normal lexical selection and the causes of the TOT experience.

\section{REFERENCES}

Bock, J. K. (1987). Coordinating words and syntax in speech plans. In A. Ellis (Ed.), Progress in the psychology of language (pp. 337390). London: Erlbaum.

BrenNen, T., B AGULEY, T., Bright, J., \& BrUCE, V. (1990). Resolving semantically induced tip-of-the-tongue states for proper nouns. Memory \& Cognition, 18, 339-347.

Brown, A. S. (1991). A review of the tip-of-the-tongue experience. Psychological Bulletin, 109, 204-223.

Brown, R., \& MeNeILL, D. (1966). The "tip of the tongue" phenomenon. Joumal of Verbal Leaming \& Verbal Behavior, 5, 325-337.

Burke, D. M., Mackay, D. G., Worthley, J. S., \& Wade, E. (1991). On the tip of the tongue: What causes word finding failures in young and older adults? Journal of Memory \& Language, 30 , 542-579.

Burnage, A. G. (1990). Celex: A guide for users. Nijmegen, The Netherlands: University of Nijmegen.
Coluss, A. M., Lortus, E. F. (1975). A spreading-activation theory of semantic processing. Psychological Review, 82, 407-428.

DeLL, G. S. (1986). A spreading-activation theory of retrieval in sentence production. Psychological Review, 93, 283-321.

Freedman, J. L., \& LANDAUER, T. K. (1966). Retrieval of long-term memory: "Tip-of-the-tongue" phenomenon. Psychonomic Science, 4, 309-310.

Garrett, M. F. (1988). Processes in language production. In F. J. Newmeyer (Ed.), Linguistics: The Cambridge survey: III. Language: Psychological and biological aspects (pp. 69-96). Cambridge: Cambridge University Press.

Grainger, J. (1990). Word frequency and neighborhood frequency effects in lexical decision and naming. Joumal of Memory \& Language, 29, 228-244

Gruneberg, M. M., Monks, J. (1974). "Feeling of knowing" and cued recall. Acta Psychologica, 38, 257-265.

JoNEs, G. V. (1989). Back to Woodworth: Role of interlopers in the tip-of-the-tongue phenomenon. Memory \& Cognition, 17, 69-76.

Jones, G. V., LANGford, S. (1987). Phonological blocking in the tip of the tongue state. Cognition, 26, 115-122.

JoNIDES, J., MACK, R. (1984). On the cost and benefit of cost and benefit. Psychological Bulletin, 96, 29-44.

KIRK, R. E. (1968). Experimental design: Procedures for the behavioral sciences. Belmont, CA: Brooks/Cole.

KozLowskı, L. T. (1977). Effects of distorted auditory and of rhyming cues on retrieval of tip-of-the-tongue words by poets and nonpoets. Memory \& Cognition, 5, 477-481.

LEVELT, W. J. M. (1989). Speaking: From intention to articulation. Cambridge, MA: MIT Press.

MACKAY, D. G. (1987). The organization of perception and action. New York: Springer-Verlag.

MAYLoR, E. A. (1990). Age, blocking and the tip of the tongue state. British Journal of Psychology, 81, 123-134.

Pease, D. M., \& Goodglass, H. (1978). The effects of cuing on picture naming in aphasia. Cortex, 14, 178-189.

RATcliff, R., McKoon, G. (1988). A retrieval theory of priming in memory. Psychological Review, 95, 385-408.

ReAson, J., \&uCAS, D. (1983). Using cognitive diaries to investigate naturally occurring memory blocks. In J. E. Harris \& P. E. Morris (Eds.), Everyday memory, actions and absent-mindedness (pp. 5370). London: Academic Press.

Roget's International Thesaurus (4th ed.) (1977). London: Harper \& Row.

Roget's Thesaurus of English Words and Phrases. (1982). Harlow, U.K.: Longman Group.

RuBin, D. C., Wallace, W. T. (1989). Rhyme and reason: Analyses of dual retrieval cues. Journal of Experimental Psychology: Leaming, Memory, \& Cognition, 15, 698-709.

STEMBerGer, J. P. (1985). An interactive activation model of language production. In A. Ellis (Ed.), Progress in the psychology of language (pp. 143-186). London: Erlbaum.

Webster's Collegiate Thesaurus. (1976). Springfield, MA: G \& C Merriam.

Webster's New Collegiate Dictionary. (1981). Springfield, MA: G \& C Merriam.

WOODWORTH, R. S. (1929). Experimental psychology. New York: Holt.

WOODWORTH, R. S. (1938). Experimental psychology. New York: Holt.

YANIV, I., MEYER, D. E. (1987). Activation and metacognition of inaccessible stored information: Potential bases for incubation effects in problem solving. Joumal of Experimental Psychology: Leaming, Memory, \& Cognition, 13, 187-205.

\section{NOTES}

1. We did not do this in Experiment 3, in which a different group of words appeared in each cuing condition.

2. One of the reviewers pointed out that this wording could engender a facilitatory processing mode. 


\section{APPENDIX A}

Materials Used in Experiments 1-3

\section{Target definition}

To keep eggs warm until hatching ${ }^{\mathrm{U}}$ Of, on, or with two sides ${ }^{B}$

Hollow or, as regards people, unintelligent, expressionless

Sparing, moderate, not self-indulgent, especially in food and drink ${ }^{P}$

Long close garment worn particularly by clergy and choir singers [choristers], often under a surplice ${ }^{P}$

Something out of keeping with the times in which it exists ${ }^{\mathrm{B}}$

To make calm or serene or to reduce agitation, especially by use of a drug ${ }^{\mathrm{U}}$

Adherent of [to] the view that human action is not free but directed by external forces acting on the will ${ }^{P}$

Able to read and write ${ }^{B}$

The desire to do evil, or for others to have ill fortune ${ }^{s}$

The study of the developing fetus before birth ${ }^{\mathrm{U}}$

Feeding on both plants and flesh

Female spirit whose wail portends death $^{\mathrm{S}}$

Referring to a phrase or way of expression natural or peculiar to a particular language ${ }^{P}$

To listen [someone who listens] to other people's conversations $\mathbf{s}$

Term describing an issue that is open to discussion or modification ${ }^{\mathrm{U}}$

Saying little, reserved, uncommunicative ${ }^{\mathrm{s}}$

Very small in size, as in "Gulliver's Travels"s

The complete remains of a dead animal, especially at a butcher's $s$

Like a beautiful, angelic child $\mathrm{U}$

State of mind in which one takes or treats things lightly or with a lack of respect $^{\mathrm{B}}$

Stoppered glass vessel in which spirits are brought to the table $\mathrm{s}^{\mathrm{s}}$

Common term for injury to the neck caused by a sudden jerk of the head, for example in $a$ vehicle collision $\mathrm{U}$

Adherent of the view that whether God exists is unknowns

$A$ young goose ${ }^{B}$

A person unnecessarily anxious about their health ${ }^{P}$

Medieval forerunner of chemistry ${ }^{P}$

To [stupefy,] bewilder or confuse ${ }^{B}$

Target

\section{Semantic}

Cue

Materials for Experiments 1 and 2

$\begin{array}{ll}\text { incubate } & \text { nurture } \\ \text { bilateral } & \text { double } \\ \text { vacuous } & \text { blank }\end{array}$

abstemious

forbearing

cassock

robe

premature

soothe

fatalist

intellectual

literate

malevolence

hostile

embryology

cellular

omnivorous

vegetarian

banshee

idiomatic

eavesdrop[per]

negotiable

taciturn
lilliputian

carcass

cherubic

flippant[cy]

decanter

whiplash

agnostic

gosling

hypochondriac

alchemy

bemuse

ghoul

euphemism

viable

withdrawn

petite

bones

sarcastic

carafe*

noose

pelican
Phonological

Cue

ignorant

bewilderment

victory

acknowledgment

careless

view

deep

hall

diminutive

lavender

mind

molecular

drink

night

head

electricity

optometrist

cow

background

imagination

seven

note

nobility

pass

tolerance

baby

legislation

comedy

conjure

wine

chihuahua

law

licker

house

depression

smile

whisper

democracy

adhesive

year

goblet

beard

hemispherical*

flute

accurate

dish

bouquet

glass 
APPENDIX A (Continued)

\begin{tabular}{|c|c|c|c|c|}
\hline Target definition & Target & $\begin{array}{c}\text { Semantic } \\
\text { Cue }\end{array}$ & $\begin{array}{l}\text { Phonological } \\
\text { Cue }\end{array}$ & $\begin{array}{c}\text { Unrelated } \\
\text { Cue }\end{array}$ \\
\hline $\begin{array}{l}\text { The escape of blood from vessels, } \\
\text { including internal as well as external } \\
\text { bleeding }\end{array}$ & hemorrhage & anemia & hurricane & window \\
\hline $\begin{array}{l}\text { Game like hockey but with ball caught } \\
\text { by, carried in, and thrown from a net } \\
\text { on a stick }\end{array}$ & lacrosse & badminton & latrine & wife \\
\hline Lover of books ${ }^{P}$ & bibliophile & reader & barbarism & heart \\
\hline $\begin{array}{l}\text { Descriptive term for the type of plant of } \\
\text { which the stem is not woody or per- } \\
\text { sistent, and whose leaves and roots } \\
\text { are often used for food, medicine, or } \\
\text { scent }^{\mathrm{B}}\end{array}$ & herbaceous & floral & harmonic & ill \\
\hline $\begin{array}{l}\text { To steam food, particularly meat, } \\
\text { slowly in a closed container }{ }^{P}\end{array}$ & braise & sauté & bridge & financial \\
\hline $\begin{array}{l}\text { House of rest for travelers or for the } \\
\text { terminally ill, often kept by a } \\
\text { religious order }\end{array}$ & hospice & ambulance & honey & tray \\
\hline $\begin{array}{l}\text { A musical term for a passage performed } \\
\text { with gradual decrease in loudness } \mathrm{P}\end{array}$ & diminuendo & waning & dissociation* & place \\
\hline Obvious or trite $\mathrm{s}$ & banal & commonplace & bizarre & judge \\
\hline
\end{tabular}

Additional Materials for Experiment 3

To fail [Someone who fails] to act, to pay, or to appear for judgment in court $^{B}$

Term used to describe a decision or person which is wicked or grossly unfair $^{\mathrm{B}}$

Puzzle in which one tries to piece together a broken picture ${ }^{\mathrm{U}}$

Relating to the great seas of the world ${ }^{\mathrm{U}}$

$$
\begin{aligned}
& \text { default[er] } \\
& \text { iniquitous }
\end{aligned}
$$

jigsaw

tyrannize*

Note-Deletions from Jones's (1989) target definitions and targets are bracketed; additions are italicized. * Cues retained from Jones's cue words. Condition assignments of definitions in Jones: ${ }^{\text {S Semantically related cue; }{ }^{\text {P }} \text { Phonologically related cue; }{ }^{\mathbf{B}} \text { Both }}$ semantically and phonologically related cue; ${ }^{U}$ Unrelated cue.

\section{APPENDIX B}

\section{Instructions for Experiment 1}

This is an experiment on tip-of-the-tongue experiences. When these occur, people know that they know a particular word, but cannot recall it. This frustrating feeling is embodied in the expression "It's on the tip of my tongue." We are interested in when such experiences occur, how they are resolved, and what kinds of information people can recover about the word, even when they cannot recall the word itself.

I am going to read you the definitions for 37 words. After each one, I will ask you to turn to a page in your booklet. There you will find a cue word that is sometimes related to the target word, sometimes in meaning and sometimes in sound. Read the cue word and then, if you know the target word, write it down immediately in the space provided.
Sometimes, you will not know what the target word is. But you may have a tip-of-the-tongue experience, knowing that you do know the word without being able to bring it to mind. When this happens, you should indicate it by responding "yes" to the question

Is the word on the tip of your tongue? Yes__ No

If you respond "yes," there will be two further questions to answer. These questions concern the initial letters and numbers of syllables in the word that is on the tip of your tongue. Sometimes you will know the answers to these questions and sometimes you will not, but either way, give your best estimate. When you are completely uncertain, just guess.

Please raise your hand if you have any questions. 